\title{
Schlittenprothesen: Was wissen wir wirklich?
}

\section{Ist ein defektes vorderes Kreuzband ein Risikofaktor für das Versagen einer Schlittenprothese? Die Ergebnisse der Arbeits- gruppe um A. Gerard zeigen, dass ein klinisch stabiles Knie mit defektem vorderem Kreuzband mit einer medialen Schlitten- prothese versorgt werden kann. Allerdings gilt dies nicht für alle Arten der Implantate ... \\ Gerard A et al. Unicondylar Arthroplasty in Knees With Deficient Anterior Cruciate Liga- ment. Clin Orthop Relat Res [in press]}

\section{Einleitung \\ $\nabla$}

Die Indikationen für die Implantation einer unikompartimentellen Knieendoprothese (Schlittenprothese) werden in der Regel streng gestellt. So wird beispielsweise ein intaktes vorderes Kreuzband (VKB) als wichtige Voraussetzung angesehen. In der Literatur werden im Vergleich zu Patienten mit intaktem VKB vermehrt aseptische Lockerungen der tibialen Komponente beschrieben und eine schnellere Abnutzung des bislang intakten Kompartimentes aufgrund der abnormalen Kinematik des Knies. Anhand eines Vergleichs von 2 Patientengruppen mit entweder einem defekten oder einem intakten VKB, überprüften A. Gerard et al., ob ein defektes VKB tatsächlich einen Risikofaktor für das Versagen einer Schlittenprothese darstellt.

\section{Methoden \\ $\nabla$}

Zwischen November 2000 und Juli 2008 wurden 72 Patienten (81 Knie) mit einem defekten VKB mit einer medialen Fixedbearing-Schlittenprothese versorgt. Aufgrund bereits klinisch bestehender VKBInstabilität wurden 5 Patienten mit einer VKB-Plastik versorgt und aus der Studie ausgeschlossen. Bei den übrigen 67 Patienten (76 Knie) ohne klinische präoperative VKB-Instabilität gelang für 68 Schlittenprothesen ein Follow-up (60 Patienten, $90 \%$ ): Bezogen auf die $90 \%$ erfassten Follow-ups erfolgte bei $58 \%$ ein klinisches und radiologisches Follow-up, bei 21\% konnten Informationen über einen Befra- gungsbogen erhoben werden. Bei 7\% erfolgte eine Revision mit Implantation einer Knietotalendoprothese, und in $4 \%$ der Fälle verstarb der Patient ohne Revisionseingriff. Die Prothesenstandzeit sowie die Versagerquote der Prothese wurden mit der Implantation von 706 medialen unikondylären Knieprothesen bei Patienten mit intaktem VKB verglichen, die im selben Zeitraum von selben Operateur implantiert wurden. Die Demografie der Patienten mit intaktem oder defektem VKB war vergleichbar. Alle Patienten erhielten

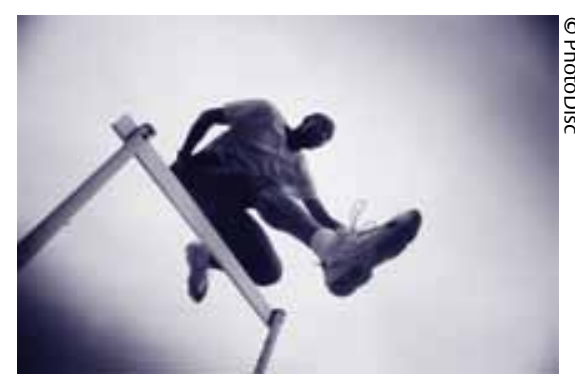

das gleiche Prothesensystem. Der durchschnittliche Nachuntersuchungszeitraum für die Patientengruppe mit defektem VKB betrug 6 Jahre (2,9-10 Jahre).

\section{Ergebnisse $\nabla$}

Es gab keinen statistisch signifikanten Unterschied $(p=0,58)$ bezüglich der Revisionsrate zwischen den Patientengruppen mit intaktem oder defektem VKB. Insgesamt 7\% der Schlittenprothesen (im Follow-up) mit defektem VKB mussten revidiert und durch eine Totalendoprothese ersetzt werden: Die Ursachen waren Progression der Arthrose im zuvor intakten Kompartiment (2 Knie), Schmerz (1 Knie), aseptische Lockerung des Tibiaplateaus (1 Knie) und Revision extern bei unbekanntem Grund (1 Knie). In der Vergleichsgruppe mussten 5\% der Schlittenprothesen revidiert werden: die Ursachen waren aseptische Lockerung (13 Knie), externe Revision bei unbekanntem Grund (11 Knie), Progression der Arthrose (3 Knie), tibiales Einsinken der Prothese (4 Knie), Infektion (3 Knie), Schmerz (1 Knie) und Überlastung des lateralen Kompartimentes (1 Knie). Die 6-Jahres-Standzeit betrug 94\% für die Schlittenprothese bei defektem VKB und $93 \%$ bei intaktem VKB $(p=0,89)$.

\section{Kommentar \\ $\nabla$}

Bisher gibt es keine eindeutig wissenschaftlich definierten Indikationen und Kontraindikationen für die Implantation einer Schlittenprothese. Allgemein gilt jedoch, dass ein intaktes VKB eine Voraussetzung für die Implantation einer Schlittenprothese ist. In der vorliegenden Studie konnten von den 68 implantierten medialen Schlittenprothesen bei gleichzeitig defizientem VKB nur 44 sowohl klinisch als auch radiologisch komplett untersucht werden (entspricht $58 \%$ der untersuchten Patienten). Über das klinische Ergebnis werden keine Angaben gemacht. Die Ergebnisse dieser Studie suggerieren, dass ein klinisch stabiles Knie mit defektem vorderem Kreuzband mit einer medialen Schlittenprothese versorgt werden könnte, ohne Nachteile bezüglich der Standzeit und Revisionsrate befürchten zu müssen. Dies gilt allerdings nur für die Verwendung eines Fixed-bearing-Implantates. Eine klinische anteroposteriore Instabilität des Knies sowie Verwendung eines Mobile-bearing-Implantates müssen weiterhin als Kontraindikation angesehen werden. Dazuhin erreicht die Studie nur ein Evidenzlevel von 3. Eine eindeutige Beurteilung und Therapieempfehlung ist daher nur begrenzt möglich. Die vorliegende Studie ist aber eine gute Grundlage zur Initiierung von prospektiven Studien mit höherem Evidenzlevel.

\section{Sarah Büermann}

Orthopädische Klinik der Medizinischen

Hochschule Hannover

sarah.bueermann@stud.mh-hannover.de 